\title{
Humoral Immune Response to Influenza Vaccination in Patients with Primary Immunoglobulin A Nephropathy

\author{
An Analysis of Isotype Distribution and Size of the Influenza-specific Antibodies
}

\author{
A. Warmold L. van den Wall Bake, * Walter E. P. Beyer, ${ }^{\ddagger}$ Jeannette H. Evers-Schouten, ${ }^{*}$ \\ Jo Hermans, Mohamed R. Daha, ${ }^{\star}$ Nic Masurel, ${ }^{\ddagger}$ and Leendert A. van Es* \\ *Department of Nephrology, University Hospital, Leiden, The Netherlands; ${ }^{\ddagger}$ Department of Virology and World Health Organization \\ Influenza Centre, Erasmus University, Rotterdam, The Netherlands; and ${ }^{\S}$ Department of Medical Statistics, \\ Leiden University, Leiden, The Netherlands
}

\begin{abstract}
Primary IgA nephropathy (IgAN) is characterized by mesangial deposits of IgA1, increased serum IgA1 levels, and circulating immune complexes containing predominantly IgA1. It has previously been found that patients with IgAN have a higher than normal IgA response to vaccination, but the IgA subclasses have not been studied. To investigate whether the IgA hyperresponsiveness is limited to the subclass IgA1, which is involved in the pathogenesis of $\operatorname{IgAN}$, we compared the immune responses of 18 patients with 22 healthy controls after intramuscular vaccination with inactivated influenza virus. Antibody titers were significantly higher $(P<0.0001)$ for the IgA1 subclass in patients versus controls, but not for the other isotypes. A substantial portion of the IgA and IgA1 antiinfluenza immune response comprised polymers in both patients and controls. There was no preferential response of polymers in patients. Patients produced significantly more monomeric IgA1 antibodies than controls. These results show that patients with IgAN have a hyperresponsiveness limited to the subclass IgA1 and mainly expressed by an excess of monomers.
\end{abstract}

\section{Introduction}

Primary IgA nephropathy (IgAN) ${ }^{1}$ has been found to be the most common form of primary glomerulonephritis in all countries where adequate epidemiological data have been acquired (1). The disease is characterized by mesangial deposits of $\operatorname{IgA}$, and there is now widespread agreement that the mesangial IgA contains almost exclusively the subclass IgA1 (2-8). Patients with IgAN have increased IgA plasma levels (4, 9-11). This increase in IgA was recently found to be due to elevated levels of IgA1 (12-14). Circulating immune complexes in IgAN also contain predominantly the subclass $\operatorname{IgA} 1(7,15,16)$. A shift in the IgA subclass distribution toward $\operatorname{IgA1}$ has re-

Address reprint requests to Dr. van den Wall Bake, Department of Nephrology, Building I C3P, University Hospital, Rijnsburgerweg 10, 2333 AA Leiden, The Netherlands.

Received for publication 29 November 1988 and in revised form 3 May 1989.

1. Abbreviations used in this paper: IgAN, primary IgA nephropathy; NBCS, newborn calf serum; VBS, Veronal-buffered saline.

J. Clin. Invest.

(c) The American Society for Clinical Investigation, Inc. 0021-9738/89/10/1070/06 \$2.00

Volume 84, October 1989, 1070-1075 cently been found in the Ig produced by peripheral blood lymphocytes (14), and in the plasma cells in the bone marrow (17) and tonsils (18) of patients with $\operatorname{IgAN}$. From these data it becomes evident that the biological abnormalities in the IgA system in patients with IgAN are predominantly or exclusively restricted to the subclass IgAl.

The pathogenetic significance of polymeric $\operatorname{IgA}$ in $\operatorname{IgAN}$ is a subject of controversy. The mesangial deposits were found to contain polymeric IgA by some investigators (7, 19-21), but not by others $(2,5,6,8)$. In the plasma, polymeric IgA was found to be elevated in two studies $(7,22)$, but no absolute $(11$, $23,24)$ or relative $(6,12,13,25,26)$ increase was found by others. These conflicting results can be partially explained by the fact that elevated serum levels of polymeric IgA are found predominantly during attacks of macroscopic hematuria (26). In recent studies predominantly monomeric IgA was found in the circulating immune complexes $(6,16,27)$.

The elevated serum levels of IgA presumably are the result of increased synthesis. A few studies have addressed the question of whether patients with IgAN have a higher than normal serum IgA immune response upon immunization. Parenteral immunization with inactivated influenza virus (28) and mumps virus (29) may lead to higher serum IgA responses in patients with IgAN. However, in the study with influenza virus no significant difference between patients and controls was reported (28). In the study with mumps virus the titers of IgAN patients were not significantly different from those in healthy controls, but they were significantly higher than the titers in patients with IgM glomerulonephritis (29). Oral immunization with polio vaccine resulted in significantly higher antigen-specific serum IgA responses in patients than in controls (30). However, none of these studies measured the subclass distribution and molecular size of the antigen-specific IgA. In view of the above-mentioned restriction of the abnormalities to the IgA1 subclass in IgAN, and the possible pathogenic role of polymers, the level and size of specific IgA1 antibodies were studied in patients with IgAN and in healthy controls after parenteral vaccination against influenza. Patients with IgAN were found to be hyperresponsive only for the IgAl isotype. However, the relative contribution of polymeric IgAl to the total amount of antigen-specific IgA1 was not different from controls.

\section{Methods}

Patients and controls. The study protocol was approved by the ethical committee of Leiden University Hospital, and all individuals gave informed consent. 18 patients ( 14 males and 4 females with a mean age of $36.0 \mathrm{yr}$; range, 24-78 yr) with biopsy-proven IgAN were studied. None of the patients had clinical or laboratory evidence of Henoch- 
Schoenlein purpura, systemic lupus erythematosus, or liver disease. Kidney function was normal in 13 patients. Mild to moderate renal insufficiency (creatinine clearance $30-84 \mathrm{ml} / \mathrm{min}$ ) was present in five patients. As controls we used 22 healthy hospital employees, 16 males and 6 females, with a mean age of $32.9 \mathrm{yr}$ (range 24-54 yr). Neither patients nor controls had clinical symptoms of mucosal infections in the 2 wk preceding the study.

Vaccination. All individuals received a single vaccination by intramuscular injection of inactivated split virus influenza vaccine containing A/Singapore/6/86 (H1N1) (Vaxigrip; Institut Mérieux, Lyon, France) within a 2-wk period in December 1986. Serum samples were obtained immediately before vaccination and at weekly intervals thereafter for a period of $5 \mathrm{wk}$. The absence of naturally occurring influenza in The Netherlands during the study period was confirmed by epidemiological monitoring. A total of 6 serum samples, from the theoretical total of 240, were not available for study. No macroscopic hematuria was noted in any of the patients during the study period.

$E L I S A$. Isotype- and subclass-specific serum antibody responses against $\mathrm{A} /$ Singapore/6/86 influenza virus were measured by ELISA. The antigen was prepared by growing $A /$ Singapore/6/86 in the allantoic cavity of embryonated eggs. The allantoic fluid was harvested, diluted 1:4 in PBS, and the virus inactivated with $0.1 \mathrm{mg} / \mathrm{ml}$ Merthiolate. The fluid was clarified by centrifugation at $2,000 \mathrm{~g}$ for $10 \mathrm{~min}$ at $4^{\circ} \mathrm{C}$. The allantoic fluid was further diluted $1: 10$ with Veronal-buffered saline (VBS), pH 7.4, containing $10 \%$ sucrose. The virus was sedimented by ultracentrifugation at $100,000 \mathrm{~g}$ for $90 \mathrm{~min}$ at $20^{\circ} \mathrm{C}$. The pellet was resuspended in VBS-sucrose using a syringe and 19-gauge needle. The ultracentrifugation step was repeated and the purified virus was stored in VBS-sucrose in small aliquots at $-70^{\circ} \mathrm{C}$. Flat-bottomed polystyrene microtiter plates (Titertek; Flow Laboratories, Zwanenburg, The Netherlands) were coated with an optimal dilution

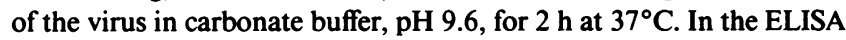
measuring the IgA subclasses, nonspecific binding sites were then blocked by incubation with PBS containing $0.05 \%$ Tween 20 and $5 \%$ heat-inactivated newborn calf serum (NBCS) for $2 \mathrm{~h}$ at $37^{\circ} \mathrm{C}$. The plates were washed with PBS-Tween three times. Samples were diluted in PBS-Tween containing 1\% NBCS and incubated in the virus-coated wells overnight at room temperature. After three washes with PBSTween, bound antibodies were detected with isotype-specific murine MAbs applied for $2 \mathrm{~h}$ at $37^{\circ} \mathrm{C}$. For the detection of IgM we used the biotin-conjugated MAb HB57, and for IgG we used the biotin-conjugated MAb HB43 (American Type Culture Collection, Rockville, MD). IgA was detected with a biotin conjugate of the MAb NI 69 (Nordic Immunological Laboratories, Tilburg, The Netherlands). IgA subclasses were detected with the MAbs NI 69-11 (anti-IgA1) and NI 512 (anti-IgA2) (Nordic Immunological Laboratories), followed by biotin-coupled goat anti-mouse IgG. After three washes with PBSTween, streptavidine conjugated to horseradish peroxidase (Zymed, Sanbio B.V., Uden, The Netherlands) in PBS-Tween-NBCS was applied to the wells for $1 \mathrm{~h}$ at $37^{\circ} \mathrm{C}$. The wells were washed three times with PBS-Tween, and substrate (o-phenylenediamine; Sigma Chemical Co., St. Louis, MO) conversion was read at $492 \mathrm{~nm}$ according to conventional methods.

All antiinfluenza titers are expressed in arbitrary units per milliliter, relative to a standard serum yielding high OD values in the ELISA for a certain isotype. A different standard serum was chosen for each isotype, and this standard serum was used in a dilution series on each ELISA plate. The standard sera yielded increasing OD values in a dose-dependent fashion to values of at least 1.000 , and usually $>2.000$. For the isotypes IgM, IgG, and IgA, the titer assigned to the standard serum was $1,000 \mathrm{U} / \mathrm{ml}$. The two IgA subclasses were measured relative to a single strongly positive serum with arbitrary titers of $8,000 \mathrm{U} / \mathrm{ml}$ of $\mathrm{IgA} 1$ and $2,000 \mathrm{U} / \mathrm{ml}$ of IgA2. Sample titers were calculated from the standard curves thus obtained. Blank values, obtained on each plate using PBS-Tween-NBCS, were usually $<0.050$, and were subtracted from the $O D$ values of standards and samples. Calculations were made at sample dilutions yielding an OD in the lower steep part of the curve.
To exclude an artifact in the IgAl antiinfluenza ELISA due to the possible presence of IgA1 rheumatoid factor in the sera, as has been reported by other investigators (16), we performed a control experiment using absorption to immobilized IgG. The sera obtained on days 0 and 14 were diluted 1:5 in PBS-Tween-NBCS, and a 500- $\mu$ l sample was incubated for $1 \mathrm{~h}$ at room temperature with $1 \mathrm{ml}$ (packed volume) of an immunoabsorbent of human IgG coupled to Biogel A5 (Bio-Rad Laboratories B.V., Utrecht, The Netherlands). For comparison, the diluted sera were incubated in a parallel fashion with a control immunoabsorbent. After separation of the absorbed samples from the immunoabsorbent by centrifugation the samples were tested for IgA 1 antiinfluenza in the ELISA mentioned above. Separate experiments demonstrated that the absorption technique used was capable of consistently removing $>95 \%$ of the IgAl rheumatoid factor from two strongly positive sera from patients with rheumatoid arthritis. The titer of IgAl antiinfluenza antibodies was not reduced in either the patients or the controls when comparing the IgG absorption with the control absorption. This demonstrates that the presence of IgAl rheumatoid factor does not influence the results in the IgA 1 antiinfluenza ELISA in any significant way.

$H P L C$. The size distribution of the influenza-specific IgA was studied using molecular sieving HPLC. The sera obtained on days 14 and 28 were diluted $1: 20$ with $0.1 \mathrm{M}$ phosphate buffer with $0.1 \mathrm{M} \mathrm{NaCl}$ (pH 6.8) and passed through a $0.22-\mu \mathrm{m}$ filter (Millipore/Continental Water Systems, Bedford, MA). $200 \mu \mathrm{l}$ of each sample was then injected onto a TSK G3000 SW column connected via a guard column to an HPLC pump (LKB, Bromma, Sweden). The column was equilibrated and run in $0.1 \mathrm{M}$ phosphate buffer with $0.1 \mathrm{M} \mathrm{NaCl}, \mathrm{pH} 6.8$, at a flow rate of $0.3 \mathrm{ml} / \mathrm{min}$. 1-min fractions were collected and assayed for total $\mathrm{IgA}$ content using the ELISA previously described (17). Based on the IgA elution profile thus obtained, fractions were pooled to form polymeric and monomeric pools for each HPLC run. The volume for each pool was determined by multiplying the number of fractions with the mean fraction volume measured in each HPLC run. In each pool the titer of influenza-specific IgA and IgAl was determined with the ELISA described above. The amount of antigen-specific IgA2 in the HPLC pools proved to be too low for detection by the ELISA. The total number of polymeric and monomeric units in each sample was determined by multiplying the titer with the pool volume. The percentage of polymeric IgA and IgAI was calculated by dividing the polymeric units by the sum of polymeric and monomeric units. Two sera from the patient group and one control serum were not available for study. Consequently, the analysis was performed on 16 patients and 21 controls.

Data analysis. All data are expressed as arithmetic means \pm SEM. The data were analyzed by two-way analysis of variance to study the effect of both group (patient versus control) and time after vaccination. For the serum titers and the units of influenza-specific antibody in the polymeric and monomeric pools, this analysis of variance was performed on their logarithmic values to normalize the data distribution. The isotype ratios in individual sera and the percentage of polymers in the HPLC study were calculated from the nontransformed data. Statistical analysis was performed with the Statgraphics software package (STSC Inc., Rockville, MD).

\section{Results}

Isotype-specific serum immune response. The logarithmically transformed titers of IgM, IgG, IgA, IgA1, and IgA2 antiinfluenza antibodies are plotted against time after vaccination in Figs. 1 and 2. Analysis of variance revealed a significant $(P$ $<0.0001$ ) increase in the IgM antiinfluenza titer after vaccination. The IgM response, however, did not differ between patients and controls $(P=0.36)$. IgG antiinfluenza titers also increased significantly after vaccination $(P<0.0001)$, with no significant difference between patients and controls $(P=0.31)$. A significant $(P<0.0001)$ increase was also found for the $\operatorname{IgA}$ antiinfluenza titers in time, and although patients had a higher 


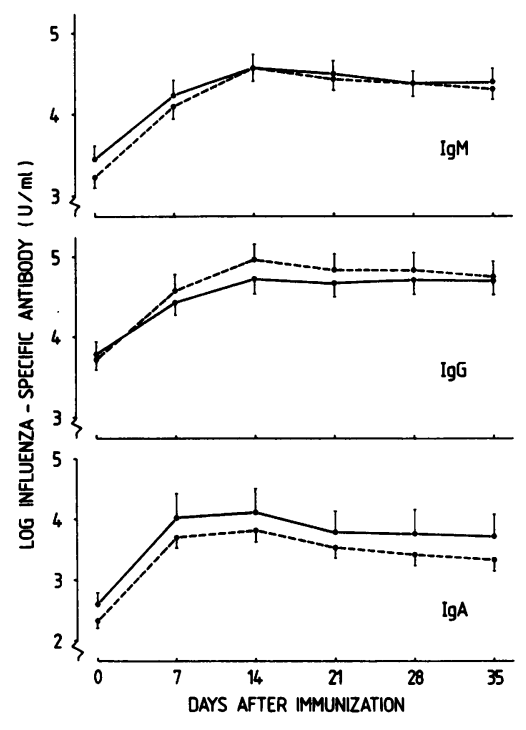

Figure 1. Plot of the IgM, IgG, and IgA antiinfluenza titers, transformed to their natural logarithms, versus time after immunization. Solid line, mean response in patients ( $n$ $=18$ ); dashed line, controls $(n=22)$; vertical bars, SEM. (Top) IgM immune response; (middle) IgG response; (bottom) IgA response. Patients were not significantly different from controls for IgM $(P$ $=0.36)$ or $\operatorname{IgG}(P=$ $0.31)$. The difference between patients and controls was marginally significant for IgA ( $P=$ 0.058).

immune response, the difference with healthy controls was not statistically significant at the 0.05 level $(P=0.058)$. The IgA1 antiinfluenza titers also increased significantly after immunization $(P=0.001)$. The IgAl immune response was markedly higher in patients than in healthy controls $(P<0.0001)$. The peak of the $\operatorname{IgA} 1$ antiinfluenza also appeared to occur earlier in patients (day 7) than in controls (day 14). For the subclass IgA2 a significant increase in the titers after immunization was also found $(P<0.0001)$, with no significant difference between the two groups $(P=0.44)$.

Isotype ratios of the immune response. The ratio of the titers of IgA and IgG antiinfluenza antibodies was calculated for each serum sample. Two-way analysis of variance demonstrated a significantly higher $(P=0.01) \mathrm{IgA} / \mathrm{IgG}$ ratio in the patients than in the controls. The influenza-specific IgA/IgG ratio did not change significantly over the weeks after vaccination $(P=0.49)$. The ratio of the $\operatorname{IgA}$ titer over the sum of the

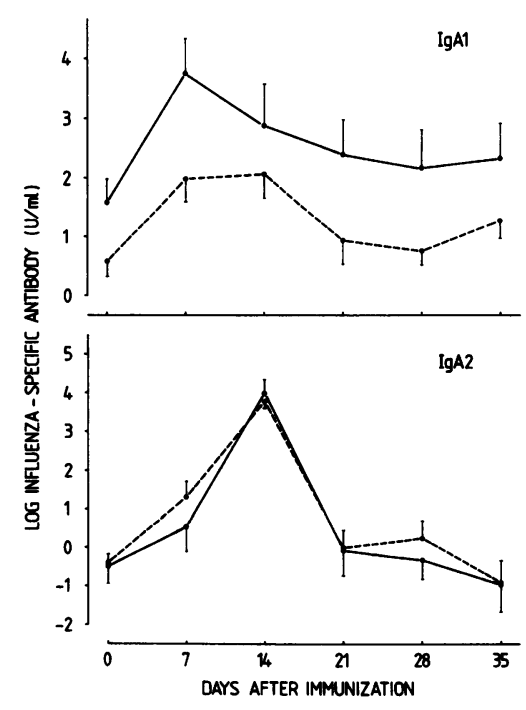

Figure 2. Plot of the IgA1 and IgA2 antiinfluenza titers, transformed to their natural logarithms, versus time after immunization. Solid line, mean response in patients $(n$ $=18$; , dashed line, controls $(n=22)$; vertical bars, SEM. (Top) IgA1 immune response; (bottom) IgA2 response. $\mathrm{Pa}$ tients had a significantly higher IgA1 antiinfluenza response than controls $(P$ $<0.0001)$. The IgA2 response was not significantly different from controls $(P=0.44)$. titers of the isotypes IgA, IgG, and IgM was also significantly higher in patients than controls $(P=0.039)$. This ratio did not vary significantly over time $(P=0.26)$.

The ratio of the IgA1 antiinfluenza titer over the sum of the IgA1 and IgA2 titers was also calculated for each serum sample. This ratio was significantly higher $(P<0.0001)$ in patients than in controls. The IgA subclass ratio also changed significantly over time after vaccination $(P<0.0001)$. This was due to the fact that the mean IgA1 response reached its peak value at an earlier time (day 7) than the mean IgA2 response (day 14), especially in the patient group.

HPLC size analysis of the influenza-specific IgA. Data from the HPLC study of the sera obtained on days 14 and 28 after vaccination are summarized in Table I. Polymeric IgA antiinfluenza was not found to be significantly different between patients and controls $(P=0.94)$ in a two-way analysis of variance. This was also the case for polymeric IgAl antiinfluenza antibodies $(P=0.19)$. Monomeric IgA antiinfluenza was not higher in patients than in controls $(P=0.28)$. However, the influenza-specific monomeric IgA 1 was significantly higher in patients with IgAN than in healthy controls $(P$ $=0.035$ ). With respect to the effects of time after immunization, the analysis of variance showed the polymeric IgA antiinfluenza to be significantly higher on day 14 than 28 ( $P$ $=0.009$ ). The differences between days 14 and 28 were not significant for polymeric IgA1 $(P=0.21)$, monomeric IgA $(P$ $=0.13)$, or monomeric $\operatorname{IgA} 1(P=0.69)$.

The relative amount of polymeric antigen-specific IgA or IgA1 was expressed as the percentage of total antigen-specific IgA or IgA1 in each serum sample. Between the two groups these percentages of polymeric IgA and IgA1 antiinfluenza did not differ $(P=0.09$ and 0.30 , respectively). The percentage of polymeric IgA and IgA1 antibodies appeared to decrease over time after immunization $(P=0.06$ for $\operatorname{IgA}$ and 0.01 for $\operatorname{IgA} 1)$.

Table I. Size Analysis of the Influenza-specific IgA and IgAI in the Sera Obtained on Days 14 and 28 after Immunization

\begin{tabular}{lccc}
\hline & Day & Patients $(n=16)$ & Controls $(n=21)$ \\
\hline Polymeric IgA antiinfluenza* & 14 & $3.48 \pm 0.27$ & $3.52 \pm 0.21$ \\
& 28 & $2.87 \pm 0.28$ & $2.78 \pm 0.26$ \\
Polymeric IgA1 & 14 & $3.91 \pm 0.29$ & $3.32 \pm 0.20$ \\
$\quad$ antiinfluenza & 28 & $3.30 \pm 0.23$ & $3.28 \pm 0.22$ \\
Monomeric IgA & 14 & $4.25 \pm 0.26$ & $4.25 \pm 0.23$ \\
antiinfluenza* & 28 & $4.17 \pm 0.29$ & $3.58 \pm 0.27$ \\
Monomeric IgA1 & 14 & $4.76 \pm 0.24$ & $4.11 \pm 0.25$ \\
antiinfluenza & 28 & $4.68 \pm 0.22$ & $4.34 \pm 0.21$ \\
\% Polymeric IgA/total IgA & 14 & $34.3 \pm 3.7$ & $35.8 \pm 3.9$ \\
& 28 & $22.7 \pm 2.2$ & $33.3 \pm 3.3$ \\
\% Polymeric IgA1/total IgA1 & 14 & $31.6 \pm 4.0$ & $32.5 \pm 2.5$ \\
& 28 & $21.7 \pm 2.5$ & $26.9 \pm 2.3$ \\
& & &
\end{tabular}

Data are presented as mean \pm SEM.

* Natural logarithm of arbitrary units of influenza-specific antibodies. ¥\% Polymeric IgA/total IgA = ratio of influenza-specific polymeric IgA to the sum of polymeric and monomeric influenza-specific IgA, expressed as percentage. 


\section{Discussion}

The present study investigated whether patients with IgAN have a selective IgA1 hyperresponsiveness that could account for the increase in the total serum IgA1 concentration (12-14), the IgAl-containing immune complexes $(7,15,16)$, and the IgA1 deposits in the mesangium (2-8) previously found. Our data, obtained after parenteral immunization with influenza vaccine, show that the IgA hyperresponsiveness previously suggested (28-30) was limited to the subclass IgA1. Patients had significantly higher titers of IgA1 antiinfluenza than controls, and the peak of the immune response was reached at an earlier time after vaccination. The IgM, IgG, and IgA2 responses were similar in patients and controls. The difference between patients and controls in the total IgA immune response to influenza virus was not statistically significant in our study $(P=0.058)$. It would be interesting to investigate whether a selective IgA1 hyperresponsiveness, similar to the findings in the present study, also occurs after a mucosal presentation of the antigen, simulating natural infections.

The study of the ratio of the antiinfluenza antibody isotypes in the sera showed a significant increase in the IgA/IgG ratio, and in the ratio of IgA to the total of the measured influenza-specific antibodies IgA, IgG, and IgM in patients. These data suggest that the hyperresponsiveness is not a general phenomenon for all isotypes, but is limited to the IgA class. Within the IgA class there was a significant shift toward the IgA1 isotype, as shown by the higher IgA1 to total IgA ratios in patients. A similar subclass shift toward IgA1 has been previously found in sera $(12,14)$, in the bone marrow $(17)$, in the tonsils (18), and in cultures of peripheral blood lymphocytes (14) from patients with IgAN. The present study shows that this subclass shift is also demonstrable in an antigen-specific study. This is an important observation, since it sheds light on the possible mechanism of the selective IgA1 increase in the sera of patients with IgAN. Theoretically, such an increase could be the result of two different mechanisms. First, patients with IgAN might be more heavily exposed, in frequency or duration, to antigens that selectively induce an IgA1 antibody response. That the IgA subclass produced may depend on the type of antigen has been shown in several studies (31-34). Another more attractive explanation is that patients with IgAN have an abnormal regulation that causes them to generate a high IgA1 response to a wide variety of antigens encountered by patients and healthy subjects alike. In view of the overall increase of the IgAl serum levels in these patients (14), it is unlikely that this hyperresponsiveness is restricted to a small number of antigens. The present study, in which an arbitrarily chosen antigen was presented to patients and controls, and which revealed a selective IgA1 hyperresponsiveness, favors the second theoretical explanation. More definite proof of the hypothesis that patients with IgAN are hyperresponders for IgA1 will require that more antigens be investigated in a similar way. Preferably, such future studies should include the use of antigens that are known to induce a predominant IgA2 immune response, such as polysaccharides (34).

For the reasons mentioned above it is likely that a disorder of immune regulation is responsible for the selective IgAl hyperresponsiveness in patients with IgAN. The mechanism of this disorder is at present unclear. Little is known about the regulation of the two subclasses of IgA, but from the available data it is likely that there is independent regulation of both subclasses (35). It is tempting to speculate about the role of ILs in this respect, since it has been recently shown that ILs are involved in the isotype regulation of the immune response (36). IL-4 and -5 may play a role in the generation of an IgA immune response (37-39), but the effect of these mediators on the relative synthesis of the IgA subclasses is at present unknown.

The pathogenetic significance of polymeric IgA in IgAN is controversial. Conflicting data have been reported on the presence of polymeric IgA in the mesangial deposits $(2,5-8$, 19-21). The present study gives no evidence that patients with IgAN preferentially produce more polymeric IgA or IgA1 antibodies in response to influenza vaccination. Rather, a signifcantly greater amount of monomeric IgA1 antiinfluenza antibodies were found. These data appear to confirm the recently reported preponderance of monomeric IgA 1 in the circulating immune complexes in $\operatorname{IgAN}(6,16,27)$. The percentage of polymeric IgA and IgA1, produced in response to influenza vaccination, is not significantly different in patients and controls. This finding is in accord with many studies of the total serum IgA and IgAl concentrations that demonstrate an increase in polymers matched by an equal or larger increase in monomers, with a resulting normal ratio of the two $(6,12,13$, $25,26)$. In bone marrow of patients with IgAN we recently have also found a normal ratio of polymeric to total IgA 1-producing plasma cells and a normal percentage of polymeric IgAl produced in cultures (40).

The considerable contribution of polymeric forms of IgA and IgAl to the total immune response in these isotypes noted in this study is not surprising in view of the recent literature on this subject. The antigen-specific IgA induced in the serum was found to be polymeric to a large extent, not only after mucosal antigen presentation $(31,33)$, but also after systemic immunization or infection $(34,41-43)$. In some investigations it was found that polymeric forms of IgA occurred especially in the early immune response, and that the percentage of polymeric antigen-specific IgA decreased at longer time intervals after the presentation of antigen $(41,42)$. A decrease in the percentage of polymeric IgA antiinfluenza in the course of time was also noted in the present study, and this decrease appeared to be more marked in the patient group.

In summary, the data indicate that patients with IgAN have a higher than normal immune response to parenterally administered inactivated influenza vaccine, limited to the IgA1 isotype. On the basis of previous studies (28-30), this hyperresponsiveness does not appear to be limited to influenza, but applies to various viral antigens. Therefore, the hyperresponsiveness seems to be polyclonal in nature. Further studies should elucidate whether this hyperresponsiveness is due to a regulatory defect in these patients.

\section{Acknowledgments}

The expert technical assistance of Mrs. M. van der Sluijs, Mrs. M. Stuiver, Mrs. C. van de Keur, Mr. R. van Dijk, and Mr. A. van der Ark is gratefully acknowledged. The authors thank Dr. J. J. Haaijman and Dr. J. Radl for their critical comments, and Mrs. C. J. M. van der Voort, Mrs. H. Kappelle-de Vries, and Mrs. M. L. Kluiters for their help in the preparation of the manuscript. 
This study was partially supported by grant C87-635 from the Dutch National Kidney Foundation.

\section{References}

1. D'Amico, G. 1987. The commonest glomerulonephritis in the world: IgA nephropathy. Q. J. Med. 64:709-727.

2. Conley, M. E., M. D. Cooper, and A. F. Michael. 1980. Selective deposition of Immunoglobulin Al in immunoglobulin A nephropathy, anaphylactoid purpura nephritis, and systemic lupus erythematosus. J. Clin. Invest. 66:1432-1436.

3. Tomino, Y., M. Endoh, Y. Nomoto, and H. Sakai. 1981. Immunoglobulin A1 in IgA nephropathy. N. Engl. J. Med. 305:1159-1160.

4. Hall, R. P., I. Stachura, J. Cason, T. L. Whiteside, and T. J. Lawley. 1983. IgA-containing circulating immune complexes in patients with IgA nephropathy. Am. J. Med. 74:56-63.

5. Lomax-Smith, J. D., L. A. Zabrowarny, G. S. Howarth, A. E. Seymour, and A. J. Woodroffe. 1983. The immunochemical characterization of mesangial IgA deposits. Am. J. Pathol. 113:359-364.

6. Katz, A., M. M. Newkirk, and M. H. Klein. 1984. Circulating and mesangial IgA in IgA nephropathy. Contrib. Nephrol. 40:74-79.

7. Valentijn, R. M., J. Radl, J. J. Haaijman, B. J. Vermeer, J. J. Weening, R. H. Kauffmann, M. R. Daha, and L. A. van Es. 1984. Circulating and mesangial secretory component-binding IgAl in primary IgA nephropathy. Kidney Int. 26:760-766.

8. Sato, M., H. Kojima, K. Takayama, and S. Koshikawa. 1988. Glomerular deposition of food antigens in IgA nephropathy. Clin. Exp. Immunol. 73:295-299.

9. van der Peet, J., L. Arisz, J. R. H. Brentjens, Marrink J., and $\mathrm{Ph}$. J. Hoedemaeker. 1977. The clinical course of IgA nephropathy in adults. Clin. Nephrol. 8:335-340.

10. Clarkson, A. R., A. E. Seymour, A. J. Thompson, W. D. G. Haynes, Y.-L. Chan, and B. Jackson. 1977. IgA nephropathy: a syndrome of uniform morphology, diverse clinical features and uncertain prognosis. Clin. Nephrol. 8:459-471.

11. Lesavre, Ph., M. Digeon, and J. F. Bach. 1982. Analysis of circulating IgA and detection of immune complexes in primary IgA nephropathy. Clin. Exp. Immunol. 48:61-69.

12. Delacroix, D. L., K. B. Elkon, A. P. Geubel, H. F. Hodgson, C. Dive, and J. P. Vaerman. 1983. Changes in size, subclass, and metabolic properties of serum immunoglobulin $A$ in liver diseases and in other diseases with high serum immunoglobulin A. J. Clin. Invest. 71:358-367.

13. Jones, C., N. Mermelstein, P. Kincaid-Smith, H. Powell, and D. Roberton. 1988. Quantitation of human serum polymeric IgA, IgA 1 and IgA2 immunoglobulin by enzyme immunoassay. Clin. Exp. Immunol. 72:344-349.

14. van den Wall Bake, A. W. L., M. R. Daha, A. van der Ark, P. S. Hiemstra, J. Radl, and L. A. van Es. 1988. Serum levels and in vitro production of IgA subclasses in patients with primary IgA nephropathy. Clin. Exp. Immunol. 74:115-120.

15. Tomino, Y., M. Miura, T. Suga, M. Endoh, Y. Nomoto, and H. Sakai. 1984. Detection of IgA1-dominant immune complexes in peripheral blood polymorphonuclear leukocytes by double immunofluorescence in patients with IgA nephropathy. Nephron. 37:137-139.

16. Czerkinsky, C., W. J. Koopman, S. Jackson, J. E. Collins, S. S. Crago, R. E. Schrohenloher, B. A. Julian, J. H. Galla, and J. Mestecky. 1986. Circulating immune complexes and immunoglobulin A rheumatoid factor in patients with mesangial immunoglobulin A nephropathies. J. Clin. Invest. 77:1931-1938.

17. van den Wall Bake, A. W. L., M. R. Daha, J. Radl, J. J. Haaijman, A. van der Ark, R. M. Valentijn, and L. A. van Es. 1988. The bone marrow as production site of the IgA deposited in the kidneys of patients with IgA nephropathy. Clin. Exp. Immunol. 72:321325.

18. Nagy, J., and P. Brandtzaeg. 1988. Tonsillar distribution of IgA and $\mathrm{IgG}$ immunocytes and production of IgA subclasses and $\mathrm{J}$ chain in tonsillitis vary with the presence or absence of $\operatorname{IgA}$ nephropathy. Scand. J. Immunol. 27:393-399.

19. Béné, M.-C., G. Faure, and J. Duheille. 1982. IgA nephropathy: characterization of the polymeric nature of mesangial deposits by in vitro binding of free secretory component. Clin. Exp. Immunol. 47:527-534.

20. Tomino, Y., H. Sakai, M. Miura, M. Endoh, and Y. Nomoto. 1982. Detection of polymeric IgA in glomeruli from patients with IgA nephropathy. Clin. Exp. Immunol. 49:419-425.

21. Monteiro, R. C., L. Halbwachs-Mecarelli, M. C. Roque-Barreira, L.-H. Noel, J. Berger, and P. Lesavre. 1985. Charge and size of mesangial IgA in IgA nephropathy. Kidney Int. 28:666-671.

22. Lopez Trascasa, M., J. Egido, J. Sancho, and L. Hernando. 1980. IgA glomerulonephritis (Berger's disease): evidence of high serum levels of polymeric IgA. Clin. Exp. Immunol. 42:247-254.

23. Clarkson, A. R., A. E. Seymour, A. J. Woodroffe, P. E. McKenzie, Y. L. Chan, and A. M. Wootton. 1980. Controlled trial of phenytoin therapy in IgA nephropathy. Clin. Nephrol. 13:215-218.

24. Woodroffe, A. J., A. A. Gormly, P. E. McKenzie, A. M. Wootton, A. J. Thompson, A. E. Seymour, and A. R. Clarkson. 1980. Immunologic studies in IgA nephropathy. Kidney Int. 18:366-374.

25. Newkirk, M. M., M. H. Klein, A. Katz, M. M. Fisher, and B. J. Underdown. 1983. Estimation of polymeric IgA in human serum: an assay based on binding of radiolabelled human secretory component with applications in the study of IgA nephropathy, IgA monoclonal gammopathy, and liver disease. J. Immunol. 130:1176-1181.

26. Feehally, J., T. J. Beatty, P. E. C. Brenchley, B. M. Coupes, N. P. Mallick, and R. J. Postlethwaite. 1986. Sequential study of the IgA system in relapsing IgA nephropathy. Kidney Int. 30:924-931.

27. Doi, T., K. Kanatsu, K. Sekita, H. Yoshida, H. Nagai, and Y. Hamashima. 1984. Detection of IgA class circulating immune complexes bound to anti-C3d antibody in patients with IgA nephropathy. J. Immunol. Methods. 96:95-104.

28. Endoh, M., T. Suga, M. Miura, Y. Tomino, Y. Nomoto, and H. Sakai. 1984. In vivo alteration of antibody production in patients with IgA nephropathy. Clin. Exp. Immunol. 57:564-570.

29. Pasternack, A., J. Mustonen, and P. Leinikki. 1986. Humoral immune response in patients with $\mathrm{IgA}$ and IgM glomerulonephritis. Clin. Exp. Immunol. 63:228-233.

30. Leinikki, P. O., J. Mustonen, and A. Pasternack. 1987. Immune response to oral polio vaccine in patients with IgA glomerulonephritis. Clin. Exp. Immunol. 68:33-38.

31. Brown, T. A., B. R. Murphy, J. Radl, J. J. Haaijman, and J. Mestecky. 1985. Subclass distribution and molecular form of immunoglobulin A hemagglutinin antibodies in sera and nasal secretions after experimental secondary infection with influenza $A$ virus in humans. J. Clin. Microbiol. 22:259-264.

32. Brown, T. A., and J. Mestecky. 1985. Immunoglobulin A subclass distribution of naturally occurring salivary antibodies to microbial antigens. Infect. Immun. 49:459-462.

33. Mascart-Lemone, F. O., J. R. Duchateau, J. Oosterom, J.-P. Butzler, and D. L. Delacroix. 1987. Kinetics of anti-Campylobacter jejuni monomeric and polymeric immunoglobulin A1 and A2 responses in serum during acute enteritis. J. Clin. Microbiol. 25:12531257.

34. Lue, C., A. Tarkowski, and J. Mestecky. 1988. Systemic immunization with pneumococcal polysaccharide vaccine induces a predominant IgA2 response of peripheral blood lymphocytes and increases of both serum and secretory anti-pneumococcal antibodies. $J$. Immunol. 140:3793-3800.

35. Kett, K., P. Brandtzaeg, J. Radl, and J. J. Haaijman. 1986. Different subclass distribution of IgA-producing cells in human lymphoid organs and various secretory tissues. J. Immunol. 136:36313635 .

36. Tesch, H., W. Mueller, and K. Rajewsky. 1986. Lymphokines 
regulate immunoglobulin isotype expression in an antigen-specific immune response. J. Immunol. 136:2892-2895.

37. Murray, P. D., D. T. McKenzie, S. L. Swain, and M. F. Kagnoff. 1987. Interleukin 5 and interleukin 4 produced by Peyer's patch $T$ cells selectively enhance immunoglobulin A expression. J. Immunol. 139:2669-2674.

38. Harriman, G. R., and W. Strober. 1987. Interleukin 5, a mucosal lymphokine? J. Immunol. 139:3553-3555.

39. Beagly, K. W., J. H. Eldridge, H. Kiyono, M. P. Everson, W. J. Koopman, T. Honjo, and J. R. McGhee. 1988. Recombinant murine IL-5 induces high rate IgA synthesis in cycling IgA-positive Peyer's patch B cells. J. Immunol. 141:2035-2042.

40. van den Wall Bake, A. W. L., M. R. Daha, J. J. Haaijman, J.
Radl, A. van der Ark, and L. A. van Es. 1989. Elevated production of polymeric and monomeric IgA 1 by the bone marrow in IgA nephropathy. Kidney Int. 35:1400-1404.

41. Negro Ponzi, A., C. Merlino, A. Angeretti, and R. Penna. 1985. Virus-specific polymeric immunoglobulin $A$ antibodies in serum from patients with rubella, measles, varicella, and herpes zoster virus infections. J. Clin. Microbiol. 22:505-509.

42. Mascart-Lemone, F., J. Duchateau, M. E. Conley, and D. L. Delacroix. 1987. A polymeric IgA response in serum can be produced by parenteral immunization. Immunology. 61:409-413.

43. Hiemstra, P. S., W. M. Baldwin, E. A. M. van der Voort, L. C. Paul, L. A. van Es, and M. R. Daha. 1988. Polymeric IgA antibody response to rabbit antithymocyte globulin in renal transplant recipients. Transplantation (Baltimore). 45:701-705. 